\title{
THE EAST END, THE WEST END, AND KING'S CROSS: ON CLUSTERING IN THE FOUR-PLAYER HOTELLING GAME
}

\author{
STEFFEN HUCK, WIELAND MÜLLER, and NICOLAAS J. VRIEND*
}

We study experimentally a standard four-player Hotelling location game with a uniform density of consumers and inelastic demand. The pure strategy Nash equilibrium configuration consists of two firms located at one quarter of the "linear city," and the other two at three quarters. We do not observe convergence to such an equilibrium. In our experimental data we find three clusters. Besides the direct proximity of the two equilibrium locations, this concerns the focal midpoint. Moreover, we observe that whereas this midpoint appears to become more notable over time, other focal points fade away. We explain how these observations are related to best-response dynamics, and to the fact that the players rely on best-responses in particular when they are close to the equilibrium configuration. (JEL C72, C91, D21, D43)

\section{INTRODUCTION}

Despite the popularity of simple location models in industrial economics and voting theory, in the tradition following Hotelling (1929), and despite the recent rise of the experimental method in economics, there have been only few experimental tests of such models. Brown-Kruse et al. (1993) and Brown-Kruse and Schenk (1999) study models with elastic demand, while Collins and Sherstyuk (2000) focus on the simpler casewhich we address, too-where demand is inelastic.

Collins and Sherstyuk implement a model with three agents who choose locations on a line segment with a uniform density of consumers (who, due to the assumption of inelastic demand, can also be seen as voters with one vote each). It is well known that

*We thank participants of the IAREP conference 2000 in Baden, Austria, and two anonymous referees for helpful comments. Special thanks to Silke Meiner for implementing the experimental software.

Huck: Senior Lecturer, Department of Economics, Royal Holloway, University of London, Egham, Surrey TW20 OEX, UK. Phone +44(0)1784-44-3910, Fax +44(0)1784-43-9534, E-mail s.huck@rhbnc.ac.uk

Müller: Department of Economics, Humbold University Berlin, Spandauer Str. 1, 10178 Berlin, Germany. Phone +49 30 2093-5673, Fax +49 30 2093-5727, Email wmueller@wiwi.hu-berlin.de

Vriend: Reader in Microeconomics, Department of Economics, Queen Mary, University of London, Mile End Road, London, E1 4NS, UK. Phone +44-20-7882-5081, Fax +44-20-8983-3580, E-mail n.vriend@qmw.ac.uk this game has no pure-strategy equilibrium (see Eaton and Lipsey [1975]). Normalizing the line segment to the unit interval, the unique mixed-strategy equilibrium prescribes uniform randomization over the middle two quarters for all firms (see Shaked [1982]). As it is a well-established fact that experimental subjects have difficulties in randomizing (see, for example, Rapoport and Budescu [1997]) it is not very surprising that Collins and Sherstyuk do not find strong support for the equilibrium hypothesis. Their empirical distribution of choices is M-shaped and has a considerably larger support than the equilibrium distribution.

In this study we analyze the case of four agents, with uniform consumer density and inelastic demand. As the equilibrium of this case implies that two firms locate at the edge of the first and second quarters, and the other two at the edge of the third and fourth quarters, this setup seems ideal for the investigation of various matters.

First, there is no other number of agents where the equilibrium prediction has a better chance to be valid. ${ }^{1}$ With three competitors, the unique symmetric equilibrium is mixed; with five the unique equilibrium configuration is asymmetric and implies unequal payoffs; and with six and more agents the equilibrium configurations cease trivial.

1. Apart from two, where equilibrium play seems 
to be unique. Thus, only the two- and the four-agent cases yield unique pure and symmetric equilibrium configurations that give identical payoffs to all agents.

Second, the equilibrium in the four-agent case has a property that makes it interesting from a behavioral and empirical point of view. Not only the focal midpoint is empty but the whole middle segment of the "linear city" is empty as well. That is, notwithstanding the nice theoretical properties, the equilibrium is not entirely intuitive and is also conflicting with casual empirical evidence. There are no cities without shops in the center, nor are there democracies without parties located in the political middle ground.

The picture we will discern in our experimental data can be summarized in the following experimental result. In the four-seller case, the equilibrium prediction is of substantial but limited help. About one-third of all choices are clustered around the equilibrium locations, but in no session do we observe convergence to equilibrium. At the same time, the focal midpoint exerts a considerable attraction with almost $10 \%$ of all choices clustered around it. Consumers profit from this considerably in the form of lower transportation costs.

The remainder of the article is organized as follows. In section II we give a theoretical account of the model we implement. In section III we present the experimental design. In section IV we analyze the results and explain the data, and section $\mathrm{V}$ concludes.

\section{THEORY}

Consider a "linear city" in which four firms produce a good at constant marginal cost. The price of the good is fixed (due to some unmodeled features of the market). Consequently, costs can be normalized to zero and the price to one. To sell the goods, the firms simultaneously choose a location on the unit interval $(0,1)$. A continuum of consumers with mass 1 is uniformly distributed along the interval. Each consumer buys 1 unit of the good. Due to (unmodeled) transportation costs, each consumer buys her good at the firm located closest to her.

As shown, for example, in Eaton and Lipsey (1975) there is a unique (purestrategy) equilibrium configuration of locations: two firms locate at one-quarter and two, the other two at three-quarters. As proved in Osborne and Pitchik (1986), there is also a symmetric mixed-strategy equilibrium where firms employ continuous and differentiable density functions that are symmetric around one-half as strategies. However, as Osborne and Pitchik (1986) illustrate, it is hard to find this equilibrium. In Appendix A we show why finding it for the four-firm case is impossible.

In an experiment a continuous model can only be approximated by a discrete model with a sufficiently fine grid. Therefore, we next derive the equilibrium solution of the fourfirm case with a finite number of locations.

Let $L=\{1,2, \ldots, X\}$ be the set of locations whose cardinality is assumed to be divisible by four, ${ }^{2}$ and let $L_{i} \in L$ be firm $i$ 's location choice. On each $j \in L$ there is one consumer who buys 1 unit from the firm located closest to her, that is, she chooses a firm $i$ such that her transportation costs $\left|j-L_{i}\right|$ are minimized. In case of more than one firm minimizing consumer $j$ 's transportation costs we assume that, if there are firms at equal distance to the left and to the right of her, she flips a coin to determine her "direction," and if she reaches a location with more than one seller she buys from each with equal probability. $^{3}$

Without loss of generality we can order the firms such that $L_{1} \leq L_{2} \leq L_{3} \leq L_{4}$. In Appendix B we prove the following.

PROPOSITION 1. In each pure-strategy equilibrium either $L_{1}=X / 4$ or $L_{1}=X / 4+1, L_{2}=$ $X / 4+1, L_{3}=3 X / 4$, and either $L_{4}=3 X / 4$ or $L_{4}=3 X / 4+1$.

Thus, there are four different (pure-strategy) equilibrium configurations; considering all possible permutations, there are 54 different equilibria. Notice that we can speak of a unique equilibrium in the sense that any equilibrium is characterized by two firms being located at the edge of the first and the second quarter of the "linear city," and the other two at the edge of the third and fourth quarter. Moreover, each equilibrium is not only

2. This makes the analysis most convenient. For different sets of locations the results are only slightly different. We omit those because we implement a number divisible by four in the experiment.

3. As explained in the instructions to the players (see Appendix C), in the experiment we actually implement an equivalent deterministic version of the consumer demand. 
FIGURE 1

The Mixed Equilibrium Probability Distribution for $X=100$

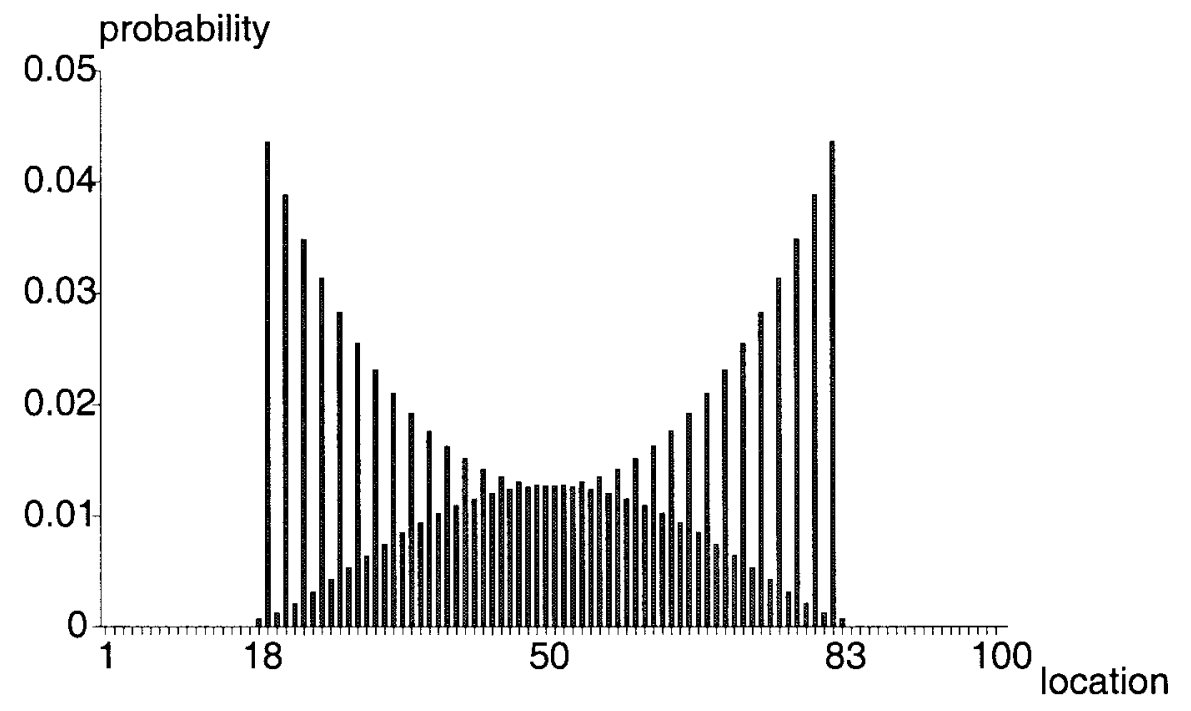

"symmetric" with respect to the midpoint of the "linear city," but is also "symmetric" as described in the following.

COROLLARY 1. In each pure-strategy equilibrium, each firm receives an identical payoff of $X / 4$.

Solving analytically for the (doubly symmetric) mixed-strategy equilibria is virtually impossible. Therefore, we employed numerical methods for different values of $X$. Figure 1 shows the equilibrium for the case of $X=100 .{ }^{4}$ The intuition behind the peculiar zigzagging shape of the equilibrium distribution is the following. First, overall the equilibrium probability distribution must be in some sense U-shaped, with the extremes of the city excluded from the support. Hence, there must be considerable mass at the left and right ends of the support. The locations outside the support are too risky, as a competitor choosing a somewhat more central location will cut a player off from most of the market, leaving only the fringes of the city, which is acceptable only if this fringe demand is large enough. For the locations in the middle of the support there is the risk that a player is

4. The expected demand for each firm is "exactly" (with 15 zeros behind the decimal point) 25 for each location in the support of the equilibrium strategy. The computer is still running for the case of $X=1,000$. squeezed in by two competitors. With a uniform distribution, this risk is too great and the expected payoffs would be lowest in the middle. Hence some probability mass must be shifted away from the center. Second, suppose a player is at the first position on the left that is played with positive probability, and she considers moving one step to the right. In equilibrium both locations have to give the same expected payoff. The benefit of moving one step to the right is small, but the potential loss is enormous because she faces the risk of seeing the complete left tail of the consumer population cut off by a rival choosing her current position. Hence to be indifferent, the probability that someone occupies this first position has to be low. Combining this with the overall U-shape gives the first zigzag step.

\section{EXPERIMENTAL DESIGN}

The computerized experiment was conducted at Humboldt University in November and December 1998. Subjects were mostly undergraduate students of economics or business administration. On arrival in the lab they were assigned a computer screen and received written instructions. ${ }^{5}$ After reading

5. For a translation of the instructions see Appendix C. 
them, questions could be asked and were answered privately. In total, six sessions consisting of 50 rounds were conducted with four participants each. The number of rounds to be played was common information. In the instructions, subjects were informed about the following. In the experiment each participant has the role of a seller who, just like three other sellers, has the opportunity to sell a fictitious product in a market. There are 1,000 simulated buyers, which are located on the integer points of a line from 1 to 1,000. The task of a seller is to choose a position (a number from 1 to 1,000) on the line with the understanding that all sellers do this simultaneously and that each consumer wishes to buy exactly 1 unit per round. Because costs are symmetric across sellers (1 Taler per unit), consumers would buy from the seller to whom the distance is shortest. (For how ties were broken see the instructions in Appendix C.)

Locations could be specified using two different methods (see Figure 2). The first method was to move an arrow to a specific position on a black line that had no ticks indicating numbers. The numerical value of the chosen location was then also shown in a small box next to the line. At the start of each round the arrow appeared at a neutral "parking position" on the right edge of the screen. The second method to enter a location was to type it directly into the aforementioned box. A click on a special icon ("Go!") then automatically moved the arrow to the corresponding position on the line. After each round a participant was informed about her

FIGURE 2

\section{A Screen Shot}

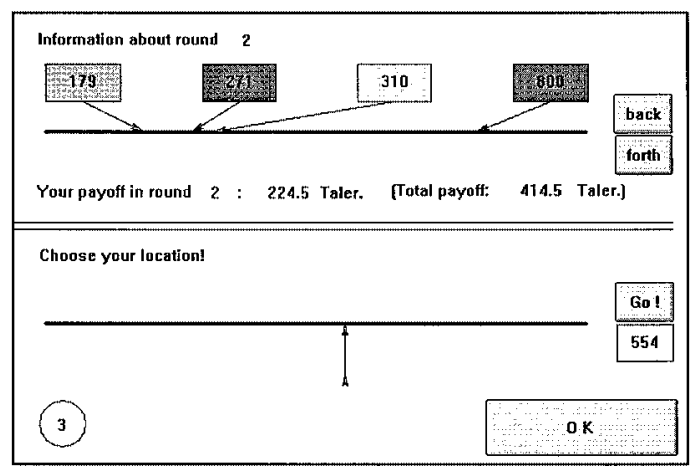

own and the positions of the other three sellers in the preceding round. This was done in horizontally arranged and colored boxes (one color for each participant). Additionally, arrows with the same colors emanating from the boxes pointed to the corresponding positions on a line below the boxes. A given player's own position was always shown in the green box (with a green arrow), whereas each of the other participants in the market was represented by one of the other colors (blue, yellow, red), which was held constant during the entire experiment. ${ }^{6}$ Each participant was informed about her own profit in the most recent round as well as about cumulative profits. Furthermore, the participants were able to recall the four positions of all preceding rounds by clicking on special icons for going back and forth.

Subjects were informed that the sum of all profits accumulated during the experiment would determine their final monetary payoff. The exchange rate was Taler $400=$ DM 1 . A session lasted between 45 and 60 minutes, and subjects' mean earnings were DM 31.25.

\section{RESULTS}

Figure 3 displays the frequency distribution of location choices across all periods (top), and for the first and second half of the experiment separately (bottom), in each case aggregated over all sessions. The eventual distribution is $\mathrm{W}$-shaped with modes at the two equilibrium locations and the focal middle. Thus, both our intuitions are confirmed. The equilibrium locations are attractive, but so is the center of the city. The three locations around which there are clusters of firms - the two equilibrium locations and the focal midpoint-in turn suggest the title of the article. ${ }^{7}$

Comparing the second half of the experiment with the first half, the following points emerge. First, there is a trend toward both

6. For instance, the player whose screen is shown in Figure 2 chose location 179 in round 2, which implied a payoff of 224.5 Taler in that round. In round 3 he considers choosing location 554.

7. King's Cross does not only correspond to Schelling's focal Grand Central, it also came out on top in an undergraduate class room game at Queen Mary presenting a London Underground variant of Schelling's coordination problem. 
FIGURE 3

Frequency Distribution of Locations

\section{Distribution of Locations - All Data}

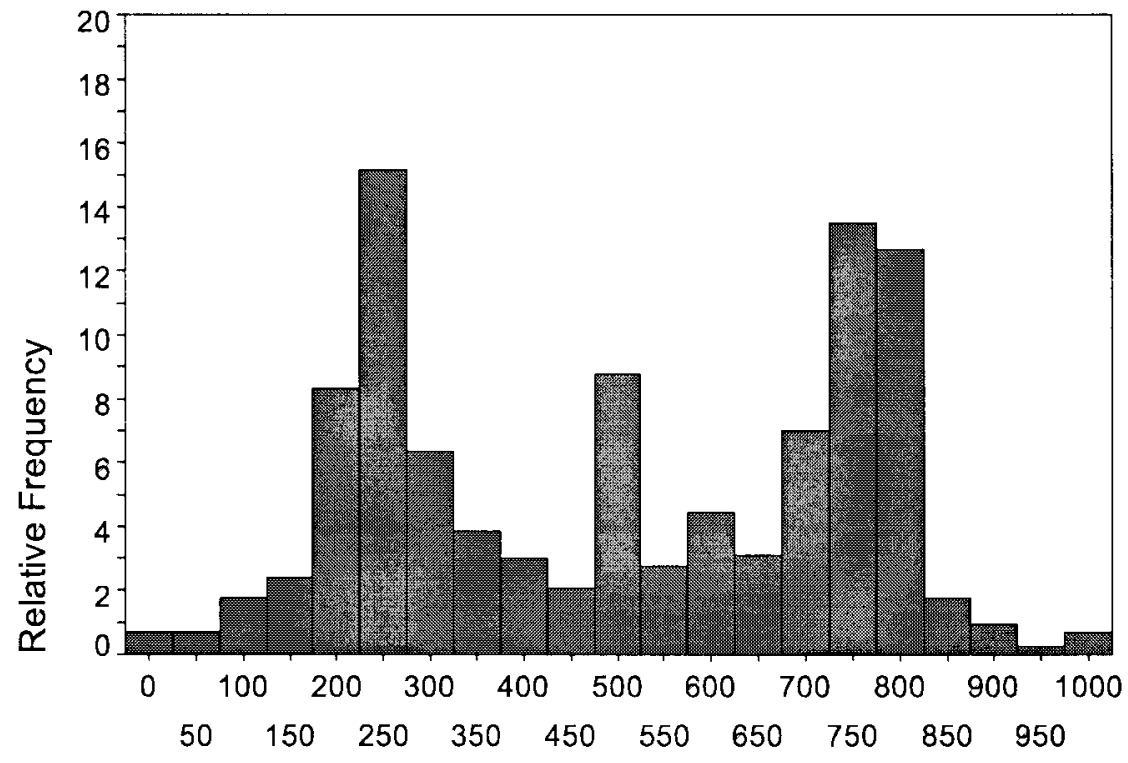

Location

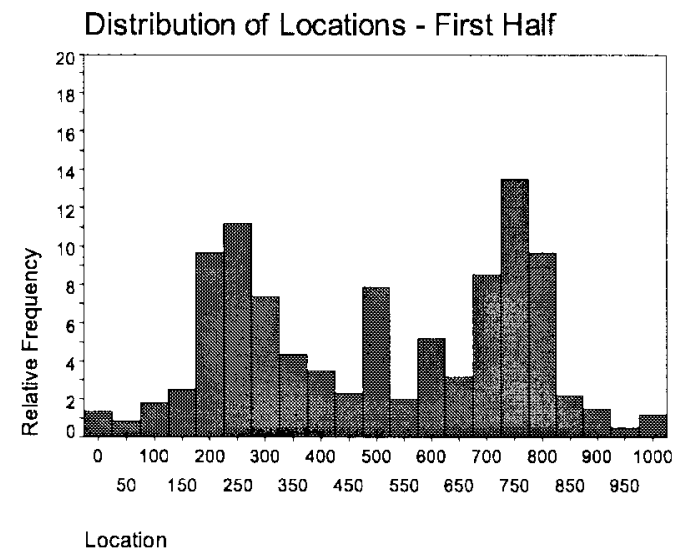

more equilibrium behavior and more clustering in the middle, and there is no real convergence to the equilibrium. Considering deviations up to a distance of 25 still to be in a location's neighborhood, we find that $19.2 \%$ of all choices in the second half of the experiment are clustered around the "western" equilibrium location, $13.5 \%$ around the "eastern" equilibrium location, and 9.7\%

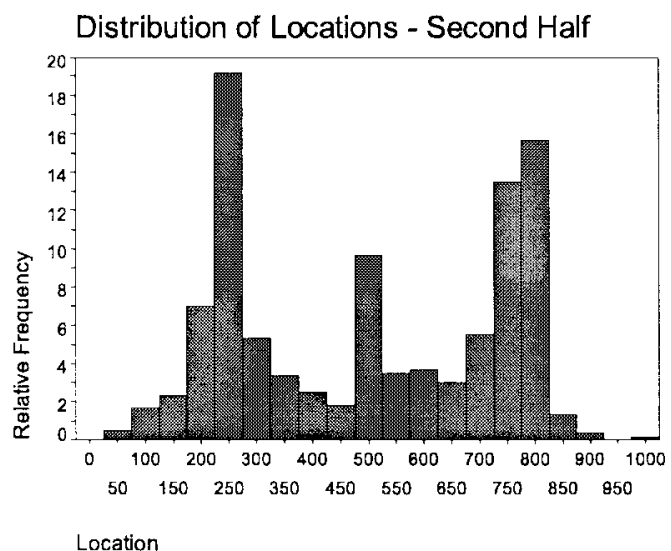

around the midpoint. ${ }^{8}$ Second, whereas the importance of the focal midpoint increases, other focal points (most notably 400 and 600, but basically all multiples of 100) actually fade away. Our investigation of the experi-

8. Notice that the lower frequency around the "eastern" location is in part explained by a slight overshooting toward the "eastern" city limit. 


\section{FIGURE 4}

The Average Distance from Equilibrium per Player for Each of the Six Sessions

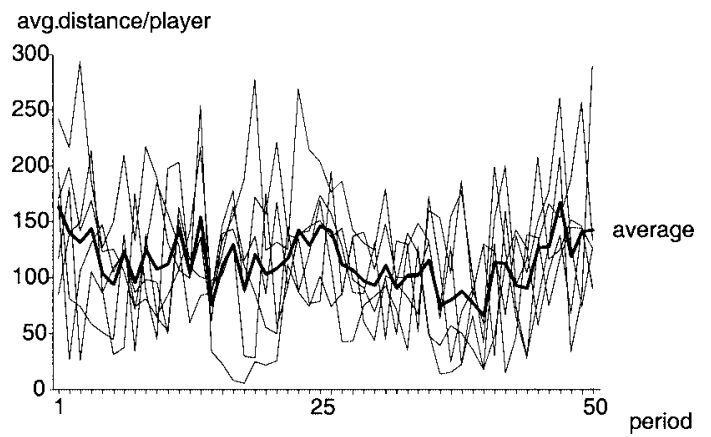

mental data will be focused on analyzing and explaining these two points.

With respect to the lack of convergence to equilibrium, it is interesting to note that there is not even a slow trend toward an equilibrium configuration. For each session we determined the distance from equilibrium in a given period as follows. We ordered the players such that $L_{1} \leq L_{2} \leq L_{3} \leq L_{4}$, and computed the average distance per player in a given period as $D=\left(\left|L_{1}-250\right|+\left|L_{2}-251\right|+\right.$ $\left.\left|L_{3}-750\right|+\left|L_{4}-751\right|\right) / 4$.

Figure 4 shows the time series of the average distance from equilibrium per player in each of the six sessions, plus the same variable averaged over the six sessions. The average $D$ over all data is 114.2 (120.6 in the first half, 107.8 in the second half of the experiment). Three out of six sessions had a higher average distance in the second half of the experiment. Overall, the difference is statistically insignificant. ${ }^{\text {}}$ We see that the average distance per player fluctuates a lot, mainly between 50 and $200 .^{10}$ Notice that in most sessions the players are occasionally very close to equilibrium but then tend to move away from it again.

The implications for social welfare are interesting. As firms are more evenly spread

9. An alternative measure for the distance from equilibrium would be the standard deviation of profits, high standard deviations indicating a large distance from equilibrium (where it is zero). In the first half of the experiments this standard deviation is on average 121.1, and in the second half 113.5 , a difference that is not significant.

10. If all players were located in the center, the average distance would be 250 . out than predicted by the equilibrium, consumers' transportation costs are lower (the sum of the firms' profits is, by assumption, constant). In equilibrium, the average travel distance would be 125 . In our data, however, we observe that this number equals 107.4 in the first half and 105.2 in the second half (with standard deviations of 35.5 and 34.7, respectively).

Why, then, do firms not converge to the equilibrium outlined in Proposition 1? One answer could be that firms actually do play an equilibrium, namely, the mixed one. But, as we know from above, the mixed-equilibrium probability distribution of location choices is U-shaped, not W-shaped with a cluster in the center. Hence, mixing as such cannot fully explain our data. It seems clear that the middle mode is due to the attractiveness of focal King's Cross. But being focal as such is of limited help in explaining our data. This is not so much because a focal-point explanation is, of course, outside the domain of standard rationality, but rather because the prominence of the focal midpoint seems to augment over time, whereas other focal points fade away.

The factor explaining both phenomena (the lack of convergence to equilibrium, and the different relevance of various focal points) is the players' use of myopic best replies. ${ }^{11}$ The pure-strategy equilibria are not stable under best-reply dynamics. What is more, best-reply dynamics lead toward the center. This does not only hold when all players adhere to best-responses but also in the following, looser sense. Best-response sets typically include a range around the center (even in most equilibria), but not the fringes of the city (the most extreme locations that could be a best-response in equilibrium are $X / 4$ and $3 X / 4+1)$. Moreover, when one player moves toward the middle, subsequent best-reply adjustments imply that the other players are dragged toward the middle as well, where they tend to cut off the earlier mover from large sections of the consumer population.

11. Here myopic means that each player assumes that the strategies chosen by the other players in the next round will be the same as the ones in the previous round. Thus, players are assumed to be myopic in the sense that they do not anticipate that the play of rivals may change from one round to another. 
As an illustration, consider the following example. Suppose the firms are currently in equilibrium, with one firm at location 250, one at 251, and the other two at 750 and 751. Now imagine the firm at 251 deviating from its equilibrium location to, say, the midpoint at 500. It does not lose, as all locations between the equilibrium locations are included in its best-reply set. In the next period, the firm that remained in the West End will relocate next to the firm in the center, which deviated first. The firm at 750 may jump over to either the West End at 249 or the East End at 752. In the next periods, further best-reply adjustments imply that within two or three periods after the initial deviation all firms are located within a distance of two or three from the midpoint. Hence, a single best-response deviation from the equilibrium (which is not irrational, as it gives the same payoff as the equilibrium payoff) does not only lead away from the equilibrium, it also induces other firms to move toward the center of the city.

An analysis of individual relocations shows that subjects pay considerable attention to (myopic) best-replies. Some $17.5 \%$ adjustments are perfect (myopic) best-replies, and $40.5 \%$ are when allowing for errors up to $10 \%$ in the payoff dimension (under the hypothesis that the other firms do not move). Moreover, the frequencies of best-replies are not declining over time (in four of the six sessions myopic best-replies are actually more frequent in the second half of the experiment).

Hence, best-replies would explain both the lack of convergence to equilibrium and the fact that the focal midpoint becomes more notable, whereas other focal points fade away. However, reasoning along this line raises the next question: If firms pay so much attention to best-replies, and given that the equilibrium is unstable under bestreply dynamics, then, why are there after all so many (approximate) equilibrium choices? The answer to this is that the degree of subjects' adherence to (myopic) best-replies is situation-dependent.

Considering all sessions and all periods, we use the median distance from equilibrium (115.5) to separate the two states "far away from" and "close to" equilibrium. Allowing for a $10 \%$ error in the payoff dimension, as previously, it turns out that players choose a best-reply in $50.0 \%$ of the cases in which the game was close to equilibrium, whereas this occurs in only $31.0 \%$ of the cases far away from equilibrium. Using these paired best-response frequencies of the six sessions as independent observations, we find that in each of the six sessions players choose bestreplies more often when the game is close to equilibrium. A sign-test shows that this is significant at 0.0156 (one-sided). Moreover, these higher frequencies of choosing bestresponses when close to equilibrium do not occur simply because the sizes of the bestresponse sets happen to be much larger in that situation. The size of the best-response sets is only very weakly negatively correlated with the distance from equilibrium. ${ }^{12}$ In other words, the players appear to have a different attitude toward best-responses dependent on the situation they face. This explains why the players are not permanently pulled away from equilibrium (through the bestresponses) and why we observe the high variation in the distances from equilibrium within each session.

The question, then, is why the players have this different attitude toward best-replies in the two states. The average gains for the four players expected from myopic best-replies in a given period are highly correlated with the distance from equilibrium. ${ }^{13}$ Although the imagined gains to be made from myopic bestreplies are based on the presumption that the other players do not move, a presumption that must be counterfactual when applied to all players, when play is close to equilibrium, this may be a reasonable first-order approximation. However, when we are far away from equilibrium and there are large potential payoff gains from best-responses looming for all players, players must be much less likely to believe that the other players will actually stay put. Hence, the players are not completely myopic. ${ }^{14}$

When far away from equilibrium, subjects obviously try to outguess each other. At this

12. The correlation coefficient is -0.08 , with a $t$ statistic of -2.56 .

13. The correlation coefficient is 0.84 (significant at $0.000)$.

14. This is with good reason, because it turns out that the total adjustments by the four players in a given period, calculating total relocations in period $t$ as $T R^{t}=$ $\Sigma_{i}\left|L_{i}^{t}-L_{i}^{t-1}\right|$, is positively correlated with the distance from equilibrium. The correlation coefficient is 0.43 with a significance level of 0.000 . 
moment we do not want to speculate which sophisticated (or perhaps simple) reasoning process the players might follow in such a situation. What drives us here is only to find an explanation as to why the players must have this different attitude toward best-responses when far away from equilibrium and when close to it, because this difference plays a key role in our explanation of the data. The key to this question is that the assumption that other players will not move (which underlies the notion of myopic best-replies) is far more realistic when play is close to equilibrium. This, in turn, drives players again away from equilibrium. The cyclic behavior of the distance from equilibrium results. So do the three modes.

\section{CONCLUSION}

Analyzing a standard four-player Hotelling game, we showed why standard rationality in the form of an equilibrium prediction is of limited help. Moreover, we also showed that a focal-point type of explanation, which is of course outside the domain of standard rationality as such, is of equally limited help.

We demonstrated how the pure-strategy Nash equilibria have the property that they are unstable under best-reply dynamics. More specifically, we illustrated how best-reply adjustments drag players toward the center of the linear city. Thus, it is the best-reply adjustments that makes the midpoint becoming more important over time, whereas other focal points fade away.

Analyzing the individual choices in the experiment we found that best-replies form an important component of the players' strategies. However, the importance of bestreplies is situation-dependent: the further firms are off the equilibrium the less likely are best-replies and vice versa. Players trust the hypothesis that others will not move much less when the current configuration is irrational. This different attitude toward bestreplies in turn explains why there is a tendency back toward equilibrium when far away from it and away from equilibrium when close to it.

On the aggregate level these movements result in a W-shaped distribution of locations. There are clusters around the equilibrium positions and the focal midpoint. Hence, we should not be surprised when, for example, democracies with four parties (as Germany used to be) do not have two left-wing and two right-wing parties.

\section{APPENDIX A: ON THE SYMMETRIC MIXED EQUILIBRIUM IN THE CONTINUOUS CASE}

As usual, let the consumers be evenly distributed along the interval $(0,1)$. A mixed strategy is a probability measure $F=F(z)$ over $(0,1)$. As in Shaked (1982) we are looking for a doubly symmetric solution suggested by the symmetric nature of the problem. Hence, we assume that $F$ is symmetric around one-half and is employed by all firms. According to a result of Dasgupta and Maskin (1986a, 1986b) $F$ is atomless. Let $f$ be the density function of $F$. The support of $f$ is assumed to be a subinterval of $[\alpha, 1-\alpha], 0 \leq \alpha<1$. Furthermore, assume that three firms choose their location according to $f$. Then, the expected payoff of the fourth firm choosing location $z \in[\alpha, 1-\alpha]$ is given by

$$
\begin{aligned}
A(z)= & 3 \int_{\alpha}^{z}(1-z+[(z-x) / 2]) f(x)(F(x))^{2} d x \\
& +6 \int_{\alpha}^{z} \int_{z}^{1-\alpha}[(y-x) / 2] f(y) f(x)(1-F(y)) d y d x \\
& +6 \int_{\alpha}^{z} \int_{z}^{1-\alpha}[(y-x) / 2] f(y) f(x) F(x) d y d x \\
& +3 \int_{z}^{1-\alpha}(z+[x-z] / 2) f(x)(1-F(x))^{2} d x .
\end{aligned}
$$

For $f$ to be an equilibrium, $A$ must be constant over the support, hence $A^{\prime}(z)=0$ for all $z \in[\alpha, 1-\alpha]$. Differentiating $A(z)$ with respect to $z$, this condition is equivalent to (see also Osborne and Pitchik [1986])

$$
\begin{aligned}
(1 / 2)[ & {[2 r f(r)(2 F(r) t(1-F(r))-1)+(1 / 3)+F(r)} \\
& \times(F(r)(1-[2 / 3](F(r))+2 f(r))-1)] \\
+ & f(r)([1 / 2]-r)(1+F(r))+f(r) \\
\times & \left(\int_{a}^{1-a} x f(x) F(x) d x-[1 / 2] r\right)=0 .
\end{aligned}
$$

It is above all the integral in this equation that makes even a numeric analysis impossible.

\section{APPENDIX B: ON PURE-STRATEGY EQUILIBRIA IN THE DISCRETE CASE}

Proof of Proposition 1: Remember that $L=\{1,2, \ldots$, $X$ \}, with $X$ being a multiple of 4 and that we order the firms such that $L_{1} \leq L_{2} \leq L_{3} \leq L_{4}$. By $\Pi_{i}$ we denote firm $i$ 's (expected) profit. Observe that $\Pi_{i} \geq \max \{X-$ $\left.L_{4}, L_{1}-1\right\}$ for all firms $i$, because otherwise firm $i$ could improve its payoff by locating itself at the outside just next to firm 1 or 4 . Because $\Sigma_{i} \Pi_{i}=X$, we get $\max \{X-$ $\left.L_{4}, L_{1}-1\right\} \leq(X / 4)$, and hence

$$
L_{1} \leq(X / 4)+1 \text { and } L_{4} \geq(3 X / 4)
$$


Next we establish the following fact:

$$
L_{2}-L_{1} \leq 1 \text { and } L_{4}-L_{3} \leq 1 .
$$

To see that (B2) holds true, suppose the opposite, that is, $L_{2}-L_{1}>1$. Then firm 1 could increase its profit by choosing $L_{1}+1$, analogously for firms 3 and 4 . Combining (1) and (2) we get

$$
L_{2} \leq(X / 4)+2 \text { and } L_{3} \geq(3 X / 4)-1 .
$$

For the moment we will focus on those cases in which the following holds: $L_{3}-L_{2}>1$. We will show later that this is the general case, and we will analyze as well the single special case where this does not hold.

Consider first $L_{1} \neq L_{2}$. Then (because otherwise firm 1 would like to relocate between firms 2 and 3) it must hold that

$$
L_{1} \geq\left(L_{3}-L_{2}\right) / 2=\left(L_{3}-L_{1}-1\right) / 2 .
$$

From (B4) we get that $L_{1} \geq\left(L_{3}-1\right) / 3$. Combining this with (B3) leads to

$$
L_{1} \geq(X / 4)-(2 / 3)
$$

Since only integer values are allowed, combining (B1) and (B5) gives $L_{1} \in\{(X / 4),(X / 4)+1\}$. Now suppose firm 1 was located at $(X / 4)+1$. Since $L_{1} \neq L_{2}$, the profit of firm 1 is greater than $(X / 4)$, and there must be at least one firm earning less than $(X / 4)$, which it could get by switching to $L_{1}-1$, that is, $(X / 4)$. Hence, we must have

$$
L_{1}=X / 4 \text { and } L_{2}=X / 4+1 .
$$

Next suppose $L_{1}=L_{2}$. Analogous to (B4), in that case it must hold that

$$
\frac{L_{3}-L_{1}-1}{4}+\frac{L_{1}}{2} \geq \frac{L_{3}-L_{1}}{2} .
$$

From (B7) it follows that $L_{1} \geq\left(L_{3}+1\right) / 3$. Combining this again with (B3) and (B1) leads to $L_{1} \in\{(X / 4),(X / 4)+$ $1\}$. Now suppose that firms 1 and 2 were located at $(X / 4)$. This is not an equilibrium because each of them would have an incentive to move to $(X / 4)+1$. Hence, we must have

$$
L_{1}=L_{2}=(X / 4)+1 .
$$

To see that $L_{3}-L_{2}>1$ is indeed the general case, observe first of all that it follows directly from (B3) whenever $X>8$. Now, suppose $X \leq 8$. If $L_{1} \neq L_{2}$ we cannot have $L_{1}=(X / 4)+1$ for the reason given above. Hence we still have $L_{3}-L_{2}>1$. From (B3) it follows that the same applies if $L_{1}=L_{2}$. The only special case arises when $X=4$. If $L_{1} \neq L_{2}$, then again we cannot get $L_{1}=(X / 4)+1$ for the same reason as above. Hence $L_{1}=(X / 4)$ and $L_{2}=(X / 4)+1$. If $L_{1}=L_{2}$, then this cannot happen at $(X / 4)$ because either $L_{3}=(3 X / 4)-1$, in which case the profit of firm 1 would be less than $(X / 4)$, which it could secure by choosing an empty location, or $L_{3} \geq(3 X / 4)$, in which case the general analysis of above goes through. Hence, it must be the case that $L_{1}=L_{2}=(X / 4)+1$.
In other words, for any $X>0$, with $X$ a multiple of 4 , all equilibrium configurations must conform to (B6) and (B8). Analogous reasoning for firms 3 and 4 leads to

$$
L_{3}=(3 X / 4) \text { and } L_{4}=(3 X / 4)+1
$$

or

$$
L_{3}=L_{4}=3 X / 4 \text {. }
$$

Having excluded all alternative configurations, the proof is completed by demonstrating that each combination of either (B6) or (B8) with either (B9) or (B10) is indeed an equilibrium configuration by checking all bestresponses.

Proof of Corollary 1: Straightforward profit calculations for each firm for each equilibrium configuration.

\section{APPENDIX C: TRANSLATION OF THE INSTRUCTIONS}

Welcome to the experiment. Please read these instructions carefully. Do not talk to your neighbors. Please keep quiet during the entire experiment. If you have any questions, please raise your hand. We will then come to your place.

In our experiment you can earn different amounts of money, depending on your behavior and that of other participants who are matched with you.

In this experiment you have the role of a seller, who supplies a fictitious product together with three other sellers on a market. You and the other three participants have the opportunity to sell the product to a group of 1,000 simulated buyers at a price of 1 Taler per unit. The buyers are located on the integer points of a line (from 1 to 1,000$)$. You and the other sellers will simultaneously choose a location (a number from 1 to 1,000) on this line. All consumers want to buy exactly 1 unit of the product. Since the product has the same price of 1 Taler at every seller, the buyers purchase the product at the seller located closest to her. Each firm sells as many units of the product as consumers come to buy there and it earns exactly this amount of money. To make it not too complicated we assume that a consumer who is located in the middle of two firms (and has by that the same distance to both of the sellers) is "split up" and buys half a unit from the left and half a unit from the right seller. In the case of more than one seller located at the same location, we assume that they serve the demand in even shares. Thereby it could happen that a fraction of a unit is sold. An example will explain this option: Assume that a consumer is located in the middle between two sellers at the same place to the left and one seller to the right, i.e., he has the same way to the two sellers on the lefthand side and to the seller on the right-hand site. Then this consumer will buy half a unit of the product from the right sellers and half a unit of the product from the left seller, i.e., both sellers to the left sell one-quarter of a unit and the seller to the right sells one-half of a unit.

The experiment consists of 50 rounds. At the beginning of each round you have to choose a location on the line. There are two methods of doing this: the first method is to move the green arrow to a specific position on the line by clicking this point on the line with the 
pointer. The green arrow is then automatically placed there. In this case the numerical value of this position is simultaneously shown in the box next to the line. The second method is to type in the position (a number from 1 and 1,000) into the box next to the line. By clicking the field "Go," the arrow moves automatically to the corresponding position on the line. Of course, you can choose a combination of both methods, e.g., determine a rough position with the arrow and choose afterward the exact position by a numerical specification. If you have finally chosen a location, confirm your decision by pressing the $\mathrm{OK}$ button. In every round you can choose an arbitrary new position or you can choose an old position again.

After each round your position and the positions of the other three participants in the preceding round are shown. They appear in boxes of different colors (one color for each participant). Additionally, arrows of the same color, emanating from the boxes, point to the corresponding position on the line. Take into account that your own position is always displayed in a green box (respectively by a green arrow). The positions of the other participants are displayed in a blue, yellow, or red box. The assignment of the colors to the other participants is constant during the entire experiment. Furthermore, you will be informed after each round about your own profit in the last round and your present accumulated sum of profits. You can find this information in the upper part of the screen.

In each round you can recall the four positions of past rounds. To do this just click on the fields labeled "back" [and] "forth" located at the upper right part of the screen.

During the entire experiment you are matched with the same three other participants who will be randomly assigned to you. Each group of four participants serve its own market.

Your final profit is determined by the sum of all profits earned during the 50 rounds. You will get for every
400 Talers 1 DM. You get your payment immediately after the experiment.

\section{REFERENCES}

Brown-Kruse, J., and D. J. Schenk. "Location, Cooperation and Communication: An Experimental Examination." International Journal of Industrial Organization, 18, 2000, 59-80.

Brown-Kruse, J., M. B. Cronshaw, and D. J. Schenk. "Theory and Experiments on Spatial Competition." Economic Inquiry, 31, 1993, 139-65.

Collins, R., and K. Sherstyuk. "Spatial Competition with Three Firms: An Experimental Study." Economic Inquiry, 38, 2000, 73-94.

Dasgupta, P., and E. Maskin. "The Existence of Equilibrium in Discontinuous Economic Games, I: Theory." Review of Economic Studies, 53, 1986a, 1-26.

"The Existence of Equilibrium in Discontinuous Economic Games, II: Applications." Review of Economic Studies, 53, 1986b, 27-41.

Eaton, B. C., and R. G. Lipsey. "The Principle of Minimum Differentiation Reconsidered: Some New Developments in the Theory of Spatial Competition." Review of Economic Studies, 42, 1975, 27-49.

Hotelling, H. "Stability in Competition." Economic Journal, 39, 1929, 41-57.

Osborne, M. J., and C. Pitchik. "The Nature of Equilibrium in a Location Model." International Economic Review, 27, 1986, 223-37.

Rapoport, A., and D. V. Budescu. "Randomization in Individual Choice Behavior." Psychological Review, 104, 1997, 603-18.

Shaked, A. "Existence and Computation of Mixed Strategy Nash Equilibrium for 3-Firms Location Problem.” Journal of Industrial Economics, 31, 1982, 93-97. 